\title{
II. Referate aus Geburtshilflich-gynäkologischen
}

\section{Gesellschaften und Vereinen}

Der Britisohe geburtshilflich-gynäkologische Kongreß, 30. Juni bis 1. Juli 1922, Liverpool, beschäftigte sich eingehend mit dem Gesamt-komplex der Eklampsie. $i^{7} / 8$ ten-London stützte sich bei seinem einführenden Referat auf das von ihm zur Verfügung gestellte Material der großen britischen Entbindungsanstalten. Unter Hinweis auf die sehr lesenswerten AusführuDgen soil hier nur hervorgehoben werden, daß die sehr weitgehende medikamentöse Behandlung der Dubliner Sehule (Rotunda Hospital) weitaus die besten Resultate für Mutter und Kind aufweist. Prophylak-tische Behandlung - Hospítalpflege - wird dringend befürwortet. Eden empfiehlt die Klassifizierung als mild und schwer; in allem soil ein Minimum geburtshilfricher Eingriffe festgehalten werden, da einfachste medikamentöse sorgsame Behandlung die besten Erfolge ergibt. In der ausgiebigen Besprechung werden Chloroform und Morphium als sehr zweifelhafte Hilfsmittel bezeichnet, Veratrum viride und Veratron von einzelnen warm befürwortet. Das Dubliner Verfahren besteht in Aus-schaltung der oralen Ernährung: nur Wasser wird gereicht, Magen- und Darmkanal ausgewaschen, die Verwendung von Morphium wird in der letzten Zeit beschränkt. Subkutane Injektionen doppelkohlensaurer Natronlösungen in die Brust, absolute Isolierung und Seitenlagerung, so daß eventuell Kopf und Schultern aus demBett znmErdboden herunter-hängen und der Speichel und Schleim aus Mund und Nase ausfließen können. Aderlaß und Kaiserschnitt werden selten angewandt. Geburts-hilfliehe Eingriffe erfolgen nur auf spezielle Indikationen. - Letzte zwei-jährige Statistik 9,09 \% Mortalität.

Amcrika ische gynäkologische Gesellschaît. 3. V. 1922. (Am. J. obst. gyn. IV. 3. S. 296.) Franklin-Maríin: Weitere Studien über Ovarientransplantation. - Polak : Welche Beziehungen bestehen zwischen Hypertension und fibroider Erkrankung des Uterus. - Clark: Ver-gleich zweier Serien von Gallenblasenerkrankungen. - Peterson: 100 Fälle pelviner Tuberkulose mit besonderer Berücksichtigung der operative!! Enderfolge. - - Williams: Gefäßdurchschnitt, der die anatomischen Ver-hältnisse eines großen Uterusmyoms aufweist.

$\mathrm{C}^{1 / 8} \mathrm{eburtshilfl}$. Gesellschaft New York. 9. V. 22. (Am. J. Obst. gyn. IY. 3. S. 313.) Caldwell: Intravenöse Verwendung von Paraldehyd bei Eklampsie. - Healy: Unheilbares Ulcus vulvae (Esthiomène) geheilt mit Proteusvakzine. - Grad: Anweisung zum Halten der Nahtfäden bei vaginalen plastischen Operationen. - Thorns: Darstellung des oberen Beckeneingangs mit XStrahlen. - Grad: Pathologie uteriner Blutungen nach 100 Fallen. Besprechung ebenda.

GeburtshiM. Gesellschaît Philadelphia. 6. IV. 1922. (Am. J. Obst. gyn. IV. 3. S. 319.) Darnell: Papilloma renis malignum. - Outerbridge Akzidendelle Uterusperforation. - Kellen: Volvulus sigmoidei nach elektivem Kaiserschnitt. In memoriam E. Everett.

Referate aus geburtshilflich-gynäkolog. Gesellscliaften u, Vere $1 / 8$ en. 351

Geburtshifl.-gyn. AM. d. New York Academy of Medicin. 23. V. 1922. (Am. J. Obst. gyn. IV. 3. S. 324.) Young: Ölgetränkte Tupfer bei Laparotomien. - Rice: Neuere Bestrebungen die Kindbettmortalität herabzusetzen. - Young: Intermittierende aspiratorische Hyperämie in der Gynäkologie. Bespr. ebenda. 
Oberrheinische Gesellschaft f. Geb. u. Gyn. in Basel vom 26. VII. 1922. Opitz: Dem Gedächtnis Senders. - Walthard: Die Resultate der Psycho-therapie beim Vaginismus. Bespr.: H. Freund, A. Mayer, Eberhard. Meyer-Ruegg. - A- Mayer: Die Zunahme der sterilen Ehen seit dem Kriege. H. Freund: Geheilte und ungeheilte Fälle von Sterilität. Bespr.: Vogí, Eberhard, Fürst, MeyerRuegg, Opitz, - Labhard: Zur Frage des Pruritus vulvae. Bespr.: Walthard, Eberhard, Opitz. A- Mayer: Über postoperative Adhäsionen in der Bauchhöhle. Bespr.: Opitz. - Vogí:

Erfahrungen mit der Praglschen Jodlösung bei Laparatomien. Bespr. : Opitz. - Fürst: Über die Indikationsstellung zur operativen und Strahlenbehandlung der Myome. Bespr.: Freund. - Opitz: Neue Erfolge unserei - Strahlenbehandlung. - Vogí: Die klinische Bedeutung der extraperitonealen Harnblasenentwicklung unter der Geburt. - Labhard: Demonstration Symphysenspalt nach Überfahren. Sect. caes. - Uterus bicornis unicollis bifores mit sagittalemBand im Scheidengewölbe. Gravid, mens. VI im rechten Horn. Abdominale Totalexstirpation. Aquarelle multipler primärer Affekte an Portio und Vulva. - Blutbilder und Kurven bei akuter myoligener Leukomie im Wochenbett. - Faustgroßes nekrotisches Myom in der Sohwangerschaft. Supravaginal angenähter gravider Uterus. III. M. - Submuköses durchgebrochenes nekrotiscbes Mjrom. - Supra- $\Lambda$ 'aginale Amputation mit extraperitonealer Stielversorgung. $\mathbf{-}-$ Aquarell eines mandelgroßen, z. T. nekrotischen Polypen an der äußeren Wand der Portio. - Opitz: Puerperaler Uterus, der noch die Plazenta enthält.

Gesellschaît í. Geburtsh. u. Gyn. in Leipzig. 30. III. 1922. Hystero-exergergie und Gravidität. Bespr.: Skuísch, Liltauer, Lichiensiein, Schweiízer: Über Uterus!; arzinom bei Sohwangerschaft. - Über Collum-karzinom bei Jugendlichen.

Belgische Gesellsehaft f. Geb. u. Gyn. 4. II. 1922. Poivin : Verknöche-rung der Tube. Bespr.: Henroiay. - Spehl: 2 Fälle von Ovarialzyste mit blutigem Inhalt, die eine schnell entwickelt bei der ersten Menstruation einer 1ßjährigen, die andere bei Stieldrehung. - Loicq: Vielkammeriger N"abelbruch (Cystadenoma papelliferum ovarie). 2. Salpingitis tuber-culosa - Crousso : Laprarena (Kapsel) Extraktinjektionen zur Verhütung postanästhetischen Erbrechens. Bespr.: Weymeersch. - Henroiay-Cheval: 2 Fälle praevia centralis. Bespr.: Weymeersch, Henroiay, Rouffarl. - 11. III. Poivin: Fibromata subperitonealia uteri gravidi. - Buggenhoui: 2 Fälle von Dystokie: Placenta praevia - abdominale Hperyotomie - enges Becken. - Van der Esi: Seltene Komplikation der Uterusretro-version: Ureterenverlagerung. - Cheval: Vaginale und intraabdominale Strahlenbehandlung wegen Collumkarzinom. -1 . I $\lambda \mathrm{r}$. Coiwelaire: Organisation von Prophylaxe und Behandlung erblicher Sjphilis in den Gebär-anstalten. Bespr.: Rou†fart, Henrotay. - Beckers: Primäre Abdominal-schwangerschaft. Bespr.: Rouffart. 